\title{
Displacement of the path of perceived movement by intersection with static contours
}

\author{
MICHAEL T. SWANSTON \\ Dundee College of Technology, Dundee, Scotland
}

\begin{abstract}
Observation of a moving dot gives rise to a perceived movement path, which has properties similar to those of real contours. If the dot crosses a line inclined to a horizontal direction of movement, it appears to undergo a vertical displacement. This displacement was found to be greatest for a line orientation of around $15^{\circ}$ with respect to the movement. At other relative orientations, the size of the perceived displacement varied in the same manner as the perceived expansion of angles formed by intersection between static contours. Movement path distortions were measured with background fields like those that produce the Hering and Zöllner illusions with continuous lines. Illusory displacements of perceived movement were found to be equivalent to the static forms. The subjective contour formed by observation of movement can therefore give rise to illusions similar to those obtained with real lines.
\end{abstract}

It is well established that the perceived orientation of a line may be altered by the proximity of another in a different orientation. This is shown by the perceived expansion of an acute angle (Carpenter \& Blakemore, 1973) and by such phenomena as the tilt illusion (e.g., Georgeson, 1973). In addition, classical geometrical illusions, such as the Hering and Zöllner figures, have been thought to be accounted for by the integration of a number of component angle expansion effects along the length of the distorted line (Berliner \& Berliner, 1948; Robinson, 1972). It has been argued that psychophysical data regarding these effects should be explained by reference to the reciprocal inhibition of orientation-selective units in the visual cortex, such as is known to occur in a variety of infra-human species. However, a number of studies have shown that orientation illusions can occur with contours that, although distinctly perceived and readily judged, have only a restricted or ambiguous physical representation. For example, the Hering illusion occurs with contours defined by a small number of dots (Coren, 1970), and the tilt illusion has been obtained with a background grating whose orientation is defined by subjective grouping of pattern elements (Swanston \& Wade, 1983). Such findings pose difficulties for neurophysiological explanations of orientation illusions, and show that subjective grouping processes can give rise to perceived contours with perceptual effects equivalent to those of continuous lines. It can be argued from this that the processes responsible for contour formation should occur prior to those responsible for orientation interactions.

In addition to these effects, the apparent direction of a moving point can be subject to illusory distortions, even

The author's mailing address is: Dundee College of Technology, Bell Street, Dundee DDI IHG, Scotland. if the speed and contrast of the point are below that needed to give rise to a contour by visual persistence. Perceived distortion of a movement path has been studied as the result of induced movement (Gogel \& Griffin, 1982; Wallach, Bacon, \& Schulman, 1978), and in the form of the "shrinking-circle illusion"' (Coren, Bradley, Hoenig, \& Girgus, 1975). Nihei $(1973,1975)$ measured a movement version of the Poggendorf illusion, in which a dot moved along the path of the usual oblique transversal, disappearing when it crossed the vertical parallels. The effect, which was named the kinetic illusion, was larger than the static form and showed a similar variation when the orientation of the parallels was changed with respect to gravity. Distortions similar to those in other geometric illusions were said to have been observed, but no measurements were reported. Fineman and Melingonis (1977) independently investigated a kinetic Poggendorf illusion, with similar results, as was also the case in comparable conditions of a study by Wenderoth and Johnson (1983). The purpose of the present study was to measure any movement path distortion with displays like those used to produce illusions of line orientation, so as to determine whether such dynamically generated subjective contours and constant real contours behave similarly.

\section{EXPERIMENT 1}

The simplest form of angle expansion arises from the intersection of two lines at an acute angle. The amount of expansion varies with the angle of intersection, reaching a maximum at around $15^{\circ}$ (Carpenter \& Blakemore, 1973). Informal observation showed that a moving dot appears to undergo a vertical displacement during intersection with a line inclined to the path of movement. Typically, the dot appeared to move towards the nearest part of the line, and then away again after the crossing, so that 
a noticeable "blip" was introduced into the movement. Similar descriptions of the effect were given by Nihei (1973) and Wenderoth and Johnson (1983), although for the rather different situation when the dot crossed the edge of the occluding Poggendorf parallels. The purpose of this experiment was to determine the change in perceived movement path as a function of the orientation of the line.

\section{Method}

Apparatus. Stimuli were presented on a 36-cm (diagonal) black-andwhite television, viewed at a distance of $114 \mathrm{~cm}$. The screen was covered by a circular mask, which subtended $8^{\circ}$ at the eye. The moving dot was a black square with sides subtending $0.2^{\circ}$ displayed on a uniform gray background. A microcomputer generated this display and moved the dot along the horizontal diameter by replotting in 1-pixel steps across the screen, which was covered by 256 pixels. The speed of movement was $4 \mathrm{deg} / \mathrm{sec}$. A transparent acetate sheet was mounted in a rotating holder in front of the screen, and a single black line, approximately $0.05^{\circ}$ in width, was drawn on this. The line was placed so as to be aligned with the diameter of the circular mask. A scale marked on the edge of the holder enabled the line to be rotated to any desired orientation.

Subjects. Eight volunteer subjects took part in the experiment.

Procedure. The subjects were required to rate any apparent vertical displacement of the dot as it crossed the inclined line. For each judgment, six crossings were observed, as the result of three back and forth traversals of the screen by the dot. Ratings were made by reference to the size of the dot, by instructing subjects to estimate vertical displacements on a scale in which the height of the dot (which was always visible) had a value of 10 . Each subject made three judgments for 20 orientations of the line. From a horizontal orientation, the line was presented in $5^{\circ}$ steps to $30^{\circ}$ both clockwise and anticlockwise. Between $30^{\circ}$ and vertical, $15^{\circ}$ steps were used, to reduce the overall number of measurements, since it was expected that any effect would be limited at these large angles. Line orientations were presented in a random order to each subject.

\section{Results and Discussion}

Each subject's results were based on the mean of the three observations at each line orientation. Figure 1 shows the mean ratings, obtained across all subjects, for displacement of perceived movement path as a function of the relative orientation of the intersected line. Ratings for equivalent line orientations clockwise and anticlockwise from the horizontal were combined, since analysis of variance showed that although there was significant variation in subjects' ratings $[\mathrm{F}(8,56)=12.08, \mathrm{p}<.01]$, there was none due to clockwise or anticlockwise relative orientation $[F(1,7)=3.49, p>.05]$ and no interaction $[F(8,56)=1.19, p>.05]$. The graph shows a marked peak at a relative orientation of around $10^{\circ}$, with a rapid decline as the angle of intersection became more acute. Increasingly large angles gave more gradual reduction in the effect, reaching zero at $90^{\circ}$. The form of this function is strikingly similar to that reported by Carpenter and Blakemore (1973) for perceived angle expansion between static lines. They found a comparable peak at about $15^{\circ}$ and a similar rate of decline with increasing and decreasing relative orientation. It can be noted that the tilt illusion, which has been interpreted in terms of inhibitory interactions between orientation detectors, also shows a maximum at a relative orientation in the region of $12^{\circ}$ (Frisby, 1979).

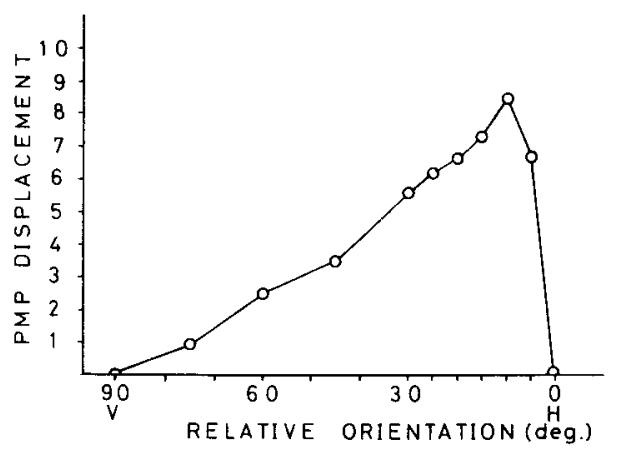

Figure 1. Mean ratings of displacement of perceived movement path (labeled PMP on the vertical axis) as a function of the relative orientation between horizontal dot movement and an inclined static line.

An estimate of the magnitude of the perceived movement path displacement can be gained from the fact that subjects made ratings based on the size of the dot, as described above. The mean maximum rating was 8.5 , which would correspond to a displacement of around $0.17^{\circ}$. This value is necessarily approximate, given the method of measurement. Although small in spatial terms, sensitivity to slight deviations from linearity of motion appears to be high, especially if, as in this case, it is rapid rather than gradual.

\section{EXPERIMENT 2}

Because the first experiment showed that a perceived movement path deviates in the region of an inclined line. there was reason to look for movement path distortions equivalent to the classical geometric illusions, such as are produced by the Hering and Zöllner figures (see Figure 2 for examples). In these illusions, a straight line is changed in orientation or curvature when crossing a field of lines at other orientations. If a perceived movement path acts

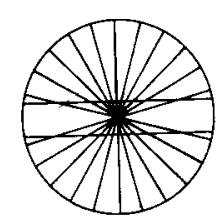

(a)

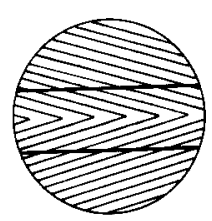

(b)

Figure 2. Examples of (a) the Hering and (b) the Zöllner illusions. The upper horizontal line in each case corresponds to the path of movement of the dot in Experiments 2 and 3. 
similarly to a real contour, then it should also undergo comparable distortions. Experiment 2 examined this for the Hering illusion. A single moving dot was employed, unlike the normal static condition, in which two parallel lines appear bowed. This was to ensure that judgments were made in terms of the change in perceived movement path rather than of relative spatial separation, as could have been the case with two moving dots.

\section{Method}

Apparatus. The apparatus was essentially the same as that described above. The inducing field consisted of radial black lines drawn on acetate, and positioned over the display screen. The dot moved along a path $0.75^{\circ}$ above the horizontal diameter of the mask at a speed of 4 $\mathrm{deg} / \mathrm{sec}$. Subjects were provided with a potentiometric joystick, whose state was sampled by the computer at each of 20 positions of the dot on its traversal of the screen.

Subjects. Four subjects took part in the experiment.

Procedure. The subjects' task was to track any changes in the vertical position of the dot by moving the joystick. They observed the dot moving through 10 traversals, making continuous adjustments of the joystick position as it did so. After these cycles, the computer displayed the mean digitized joystick setting for each of the 20 horizontal positions. This procedure was repeated three times, with the first run being discarded as practice.

\section{Results and Discussion}

Each subject's joystick settings were normalized with respect to the value recorded when the dot crossed the central vertical line of the inducing field. This provided a compensation for variation in the absolute amount of joystick movement used by each subject. Maximum deflection varied between subjects from about $15 \%$ to $25 \%$ of the total available travel. Figure 3 shows the mean settings, as a percentage of the value at the central position. The perceived movement path showed a significant displacement as the dot traversed each half of the inducing field $[F(9,27)=172.04, p<.01]$, but the displacement was not significantly different in the left and right halves of the field $[F(1,3)=0.54, p>.05]$ and there was no interaction between these two factors $[F(9,27)=$ $1.56, p>.05$ ]. Little normative information is available regarding the normal static version of this illusion, but

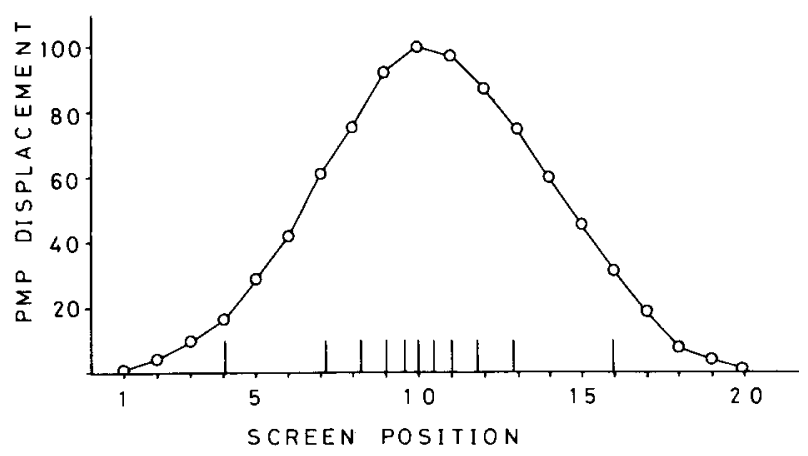

Figure 3. Perceived movement path (PMP) displacement as a function of horizontal position in Experiment 2. The short vertical lines on the horizontal axis mark the points of intersection with the radial lines of the inducing field.
Crassini and Over (1974) found that the bowing of parallel lines was due to changes in their perceived separation at the edges of the inducing field, where the intersect angle is small. In the central region, separation was judged to be the same as an undistorted control. This would be predicted if the overall illusion resulted from an integration of component angular distortions, which would be greatest when the angle of intersection was around $10^{\circ}$ to $20^{\circ}$. In the present case, subjects reported changes from the perceived movement path in the periphery of the field, but this was purely conventional, and the illusory change in perceived movement path could have occurred in this region. The results of Experiment 1 suggest that this is likely to have been the case.

\section{EXPERIMENT 3}

This experiment was designed to measure any perceived movement path distortion with an inducing field of static lines like that used to produce theZöllner illusion. As with Experiment 2, a single moving dot was presented, against a background of lines inclined at $15^{\circ}$ to the horizontal, because this gives the largest effect with static lines (Wallace \& Crampin, 1969). Two dot speeds were employed because Nihei (1973) had found that the magnitude of the kinetic Poggendorf illusion was velocity dependent.

\section{Method}

Apparatus. For this experiment, the display was generated by a microcomputer with more powerful graphics capabilities. The screen was covered by a circular mask $5^{\circ}$ in diameter, on either side of which were vertical slots. Within each of these, the position of small luminous markers could be adjusted by means of a joystick. The speed of the dot movement could be set to either 4 or $2 \mathrm{deg} / \mathrm{sec}$, and the length of the movement path subtended $4^{\circ}$ at the eye. For the illusion condition, an acetate sheet was placed behind the mask. A field of black lines was drawn on this at an orientation of $15^{\circ}$ to the horizontal. Line separation was $2.75^{\circ}$.

Subjects. Nine subjects took part.

Procedure. The subjects' task was to observe the dot moving across the screen, and to adjust the vertical position of each marker so that it was aligned with the apparent direction of travel of the dot. Their instructions were to position the markers so that the dot would contact them if it were to continue on its perceived motion path. The subjects made two settings with each of the dot speeds and with a blank backgronnd field as a control. Practice trials with each condition were given, and no subjects found any difficulty with the task. This type of alignment procedure has generally been found to be reliable for measurement of orientation illusions (Mitriani \& Yakimoff, 1983).

\section{Results and Discussion}

Scores on each trial consisted of the difference between the vertical settings of the left and right markers. To compensate for any constant error, the value obtained in the control condition with a blank background was subtracted from each of the dot speed conditions. This net score represented the perceived displacement of the perceived movement path in each case. A positive value indicated that the dot was seen to move upwards when traveling left to right, and down in the reverse direction. Given the field orientation, this was the displacement to be expected 
for a standard Hering figure. Raw scores were expressed in pixel units, since this represented the positional resolution of the display system. Each pixel covered $0.72 \mathrm{~mm}$ on the screen, and the separation between the luminous markers was $178 \mathrm{~mm}$. From this, an illusion score could be computed in terms of perceived angular rotation of the perceived movement path from the horizontal. For the speeds of 4 and $2 \mathrm{deg} / \mathrm{sec}$, the mean illusions were $+1.7^{\circ}$ and $+1.8^{\circ}$, respectively. These values were not significantly different $[\mathrm{t}(8)=0.31, \mathrm{p}>.05]$, and are somewhat lower than those reported for static versions of the Zöllner illusion with a comparable field density. For example, Wallace and Moulden (1973) reported illusory displacements of around $3.5^{\circ}$ at an intersect angle of $15^{\circ}$, and Wallace and Crampin (1969) obtained values of around $2.5^{\circ}$. This finding contrasts with those of Fineman and Melingonis (1977) and Nihei (1973), who reported larger values for the kinetic as opposed to the static version of the Poggendorf illusion.

\section{GENERAL DISCUSSION}

The experiments reported here extend the initial observations of Nihei $(1973,1975)$, and show that a slowly moving point undergoes a displacement of perceived direction as the result of intersection with static lines inclined to the direction of movement. The extent of the displacement is maximal when the static line is oriented at around $15^{\circ}$ to the movement path. The function relating displacement to relative line orientation is very similar in form to that found for perceived expansion of acute angles, which suggest that a common mechanism may be involved. The size of the movement path displacement appears to be comparable to that found with real lines and larger than has been reported with subjective contours derived from incomplete figures or arrangements of dots.

The movement path illusion with Hering and Zöllner figures parallels the form of the static version. A similar account may be given of this as has been made for the static case, in terms of the integration of small displacements occurring at each intersection. It will also be useful to look for other equivalences between the static and dynamic forms of these illusions, for example, the influence of changing the orientation of the inducing field with respect to gravity. The dynamic version offers some advantages for investigation, particularly in the case of the Hering illusion. The pattern of distortion in this illusion is relatively complex, but can be measured continuously across the field when based on displacement of perceived movement.

The results reported here have been primarily related to comparable effects with static contours. However, it is also of interest that displacements of one movement direction by another have been described by Mather and Moulden (1980). They found that if two superimposed fields of moving dots were observed simultaneously, their directions of movement were shifted away from each other. This shift was large (of the order of $12^{\circ}$ ), and declined gradually in magnitude from a relative orientation of $15^{\circ}$ to $90^{\circ}$. Relative orientations smaller than $15^{\circ}$ were not measureable, so that there was no evidence concerning the location of the maximum effect. This simultaneous repulsion of movement direction parallels the successive effect previously described by Levinson and Sekuler (1976).

It seems that static orientation and movement direction show similar effects both within and between their respective domains. The strong possibility is that there is a common mechanism underlying these interactions, and this is likely to be some form of reciprocal inhibition. ${ }^{1}$ This has been advanced by Carpenter and Blakemore (1973) to explain both static orientation effects and by Levinson and Sekuler (1976) to account for the movement interactions described above. It is therefore plausible to suggest a similar basis for the present findings. If so, there may be a link between the systems responsive to movement direction and orientation, such that each is capable of exerting a reciprocal inhibition on the activity of the other when stimulation falls within a comparable range. Alternatively, both systems might feed into a higher level of analysis, in which "direction," whether static or dynamic, is processed as a stimulus characteristic. If the latter suggestion is correct, it would account for the contourlike impression given by the path of a moving point. In either case, it is likely that further investigation of interactions between movement and static contours will shed light on the ways in which the two systems are phenomenally integrated.

\section{REFERENCES}

Berliner, A., B Berliner, S. (1948). The distortion of straight and curved lines in geometric fields. American Joumal of Psychology, 61, 153-166.

Carpenter, R. H. S., Blakemore, C. (1973). Interactions between orientations in human vision. Experimental Brain Research, 18, 287-303.

COREN, S. (1970). Lateral inhibition and the Wundt-Hering illusion. Psychonomic Science, 18, 341-342.

Coren, S., Bradley, D. R., Hoenig, P., \& Girgus, J. S. (1975). The effect of smooth tracking and saccadic eye movements on the perception of size: The shrinking circle illusion. Vision Research, 15, 49-55.

Crassini, B., \& Over, R. (1974). Loci of distortion in the Wundt and Hering illusory figures. Vision Research, 14, 1491-1492.

Fineman, M. B., \& Melingonis, M. P. (1977). The effect of a moving dot transversal on the Poggendorf illusion. Perception \& Psychophysics, 21, 153-156.

Frisby, J. P. (1979). Seeing. Oxford: Oxford University Press.

GEORGESON, M. A. (1973). Spatial frequency selectivity of a visual tilt. illusion. Nature, 245, 43-45.

GoGEL, W. C. (1977). The Wundt-Hering illusion with moving points. Bulletin of the Psychonomic Society, 10, 249. (Abstract)

GoGel, W. C., \& GrifFin, B. W. (1982). Spatial induction of illusory motion. Perception, 11, 187-189.

GoGel, W. C., \& MCNulTy, P. (1983). Perceived velocity as a function of reference mark density. Scandinavian Journal of Psychology, 24, 257-265.

LEVINSON, E., \& SEKULER, R. (1976). Adaptation alters perceived direction of motion. Vision Research, 16, 779-781.

Mather, G., Moulden, B. (1980). A simultaneous shift in apparent direction: Further evidence for a 'distribution-shift' model of direc- 
tion coding. Quarterly Journal of Experimental Psychology, 32, 325-333.

Mrtriani, L., \& YAKIMOFF, N. (1983). A model of perceived intersection of two converging line segments. Perception \& Psychophysics, 33, 186-192.

NiHEI, Y. (1973). A preliminary study on the geometrical illusion of motion path: The kinetic illusion. Tohoku Psychologica Folia, 32, 108-114.

NiHEI, Y. (1975). The effect of direction of motion on the magnitude of the geometric illusion of motion path: The kinetic illusion. Tohoku Psychologica Folia, 34, 88-94.

RoBinson, J. O. (1972). The psychology of visual illusion. London: Hutchinson.

Swanston, M. T., \& WADE, N. J. (1983). The influence of perceptual grouping on the tilt illusion. Psychological Research, 45, 107-115.

WAllace, G. K., \& Crampin, D. J. (1969). The effect of background density on the Zöllner illusion. Vision Research, 9, 167-177.

WALlace, G. K., \& Moulden, B. (1973). The effect of body tilt on the Zöllner illusion. Quarterly Joumal of Experimental Psychology, $25,10-21$.

Wallach, H., Bacon, J., \& Schulman, P. (1978). Adaptation in mo- tion perception: Alteration of induced motion. Perception \& Psychophysics, 24, 509-514.

WENDERoth, P., \& Johnson, M. (1983). Relationships between the kinetic illusion, alternating-line, and Poggendorf illusions: The effects of interstimulus interval, inducing parallels, and fixation. Perception \& Psychophysics, 34, 273-279.

\section{NOTE}

1. Gogel (1977) has also reported the occurrence of a kinetic form of the Hering illusion, but has proposed that this is due to the effect of the spatial density of the static lines on the perceived velocity of a moving point (Gogel \& McNulty, 1983). Although this explanation was not explicitly tested in the present experiments, it would not predict the effects of intersection with a single line, or the variation with its orientation as found in Experiment 1.

(Manuscript received March 19, 1984; accepted for publication July 18, 1984.) 